\title{
An introduction to the emotional impact modeling method of an event
}

\author{
Hind Zaaraoui
}

\begin{abstract}
This study develops a new way to model the emotional impact on a particular individual (person, group,...) that may have feelings, to any event or any information. The significance of an event or an information depends on the person undergoing it. Each person reacts emotionally differently to a same situation in terms of intensity and/or type of emotion. A particular same event is therefore perceived subjectively different by each individual. The reason is that each person has his own personality, his own experience, his own history, his own education and tradition ...

In this study, we assume that all the Events / Information have common interpretable points relative to each individual: Who is in the event ?, What is done? When it is done? Where it is done? How often/much it is done? Why it is done? and How it is done? Each element of the vector [Who, What, When, Where, HowMuch, Why, How] will be called as an emotional stimulus and will be mathematically modeled relatively to the studied individual. These stimuli, interpreted differently by each individual, will be the starting point for the creation of emotions at different intensities. The objective of this study is to mathematically link these different stimuli to the intensity of the resulting emotions following an event relatively to an individual.
\end{abstract}

\section{INTRODUCTION}

An emotion is a psychological, a physiological and a physical reaction to a situation [1]. It first has an internal manifestation and causes an external reaction (behavior). This is due to confronting that situation and interpreting the reality relatively to our experiences, our needs, our lifes...

Since Descartes, several theories on emotions have been developed. The initial idea was that emotion and cognition are completely independent. Emotion, created by the body and not by the mind, was something that is opposed the reflexion and the cognition.

It was only in the $80 \mathrm{~s}$, that thoughts have changed compared to the emotions. It has initially been through studies on the effects of stress that we have started to consider more seriously the possible correlation between emotional reactions and cognitive activities [2].

New theories such as the cognitive assessment which postulates that the assessment of a stimulus, event or situation by the body determines the triggering of an emotion, reinforces this new theory. Theories of cognitive assessment currently dominate the field of study on how emotions are generated and differentiated. It is recognized that the type and the intensity of the emotional experience depends on the result of the evaluation of an event and its significance to the past and personal experiences. An emotion is then a physiological answer to a stimuli or a set of stimulis according to Antonio R. Damasio and Joseph E. Ledoux ([3], [4]).

Thus one can deduce that emotions are constructed from our brain and precisely from our personal past experiences [5]. Every situation at his time has his unique personal interpretation 
and an emotional significance according to the cognitive assessment theories researchers. This evaluation is subjective, immediate and intuitive. Emotions are therefore mainly described as a cognitive process in which the physiological component occupies a minor place or is one of a resulted reaction to an emotion (see for example [6],[7],[8]). In the 60s a new theory on emotions was born with Arnold [9] and had been developped by Lazarus [10].

In this study we will suppose that an emotion is a result of the personnal relative evaluation of an event/information as assumed by some cognitive emotion theory supporters.

The rise of this emotional evaluation and prediction came with the evolution of the virtual universe including social networks, smartphone applications... where individuals can directly and rapidly interact with each other, can give his personal review, his opinion, his rating, and his feedbacks with little hindsight as they have the possibility of not being recognized in the web. Hiding behind screens and new profiles has allowed people to release (a little) their buried emotions and thus behave according more or less to their emotions. Researchers in computing and applied mathematics created a new theory to predict these "sentiments" called sentiment analysis. It uses the Natural Language Processing (NLP) which consists on text analysis and computational techniques to automate the extraction or classification of sentiment or emotions using machine learning and all technics of Artificial Intelligence (AI) from sentiment reviews. The studies were on different levels: from a general document level [11] to a word level [12] going through sentence analysis [13].

We consider an event as a moving box that may or not hit the studied person. How much will this studied person would be injured?

In the field of Physics, the analysis will not stop at the shape of the box, or how another box had struck another person in the past and identify statistically how this box will impact the person... But we will see what the box contains, what is its mass, what is the speed of the box, what is its trajectory, will it manage to reach the person before he moves, what is also the person's mass, what is the force of friction...? The assessment of the impact event on this person must be like this evaluation. What are these Physical questions that a person asks considering an approaching Event?

The physical stimuli considered in our study that create the emotion to an individual are these seven points [Who, What, Where, When, How much, Why, How], and the relative questions are What is the Who doing, When, Where, Why, and How ? and also How much is he doing ?

We hence want to model mathematically the four first stimuli [Who, What, Where, When] in this study.

In section I we describe in general how an event may create an emotion, in section II and III we model the emotional impact of the Who and the What of the events, in section IV we give an introduction on how we can model some emotions thanks to the What value. In section $\mathrm{V}$ and VI we provide the Where and the When models and finally in the last section we anamyze how the intensity of an emotion resulted from an event may evolve during time.

\section{Instantaneous EMOTIONAL IMPACT OF AN EVENT DESCRIPTION}

Emotion felt by an individual following the occurrence of an event or the arrival of information differ in type and in intensity. A same event can create different emotions between individuals 
with also different intensities, but it may also create different types of emotion in a same person in different contexts. How a same event can create different emotions?

An emotional impacting event must therefore be analyzed subjectively to the experience and the past of each individual and not on how his brain/heart is structured. The physiology of individuals plays little role in the analysis of the emotional events since a same individual may have different emotion to a same event in two different times: would I feel the same emotion if my colleague is receiving a bonus of 1 million euros for the first time or for the twentieth time?. The event "my colleague is receiving a bonus of 1 million dollar" is the same but I won't feel samely if it was the first time or for the twentieth time. Physiologically my brain in general, my heart, my DNA did not change since I heard this information for the first time and yet my feelings have changed!

Emotions created by an event are therefore an evaluation of the event in itself but also a perpetual comparison to our past live and experiences and to what we know as information and what we own.

In this section we will just analyse the emotional evaluation of an event.

But First we explain what the word Event means in our study: an Event is all that happens outside or inside of us as long as we see it, feel it, hear it, smell it ...

As presented in the introduction, the emotional impact event occured at time $T$ is defined by a function of the vector of [Who, What, Where, When, How much, Why, How]. Let the intensity evolution of the emotion $e$ until time $t$ of a particular Individual $P$ to an event occured at time $T\left(\right.$ Event $\left._{T}\right)$ defined as following:

$$
I_{e}\left(P, \text { Event }_{T}, t\right)=F_{e, P}\left(T, \text { Who }_{T}, \text { What }_{T}, \text { Where }_{T}, \text { When }_{T}, \text { How }_{\text {much }}, \text { Why }_{T}, \text { How }_{T}\right)
$$

where $F_{e, P}$ is a function which depends on the studied emotion $e$ and links [Who, What ${ }_{T}$, Where $e_{T}$, When $_{T}$, How much $_{T}, W h y_{T}, H_{o w}$ ] of the Event $_{T}$ to the evolution of the intensity of the emotion $e$ until time $t$ of the individual $P$. The evolution of an emotion is quite complexe as it has to take into account memory processes of a human and new theories of emotion variations in time.

The emotion that overwhelms the studied person $P$ at time $T$ is supposed to be the one that has the greatest intensity at time $T$. Which can be modeled as follows:

$$
\text { Emotion }_{T}=\operatorname{argmax}_{e} I_{e}\left(P, \text { Event }_{t}, T\right)
$$

In this paper the objective now is to model only the four first stimuli [Who, What, Where, When] that depend on the studied person $P$ and the emotion studied $e$.

\section{THE Who MODEL: 5 IMPACT TYPES}

The Who of the event (and not the one that is impacted) can be a person, an animal, an object, a thing, a group, a concept, an organization, a country ... The Who of the analyzed event changes the impact on the emotion felt significantly, see for example the difference between the event to me "my son leaves for the USA tomorrow" or the other event "the son of my husband's cousin's neighbor leaves for the USA tomorrow". The Who in these events, either my son or the other person, considerably affects the emotional result felt. Why and how the term "my son" is more 
impacting than "the son of my husband's cousin's neighbor". The definition of the terms here does not help us to analyze the impacts: I could not bear anymore my son with his bad behavior toward me and thus I could rejoice in his move. I could also adore my son and his move would make me miserable. I could have seen my son since his birth only very rarely and therefore his trip would not change anything, while I see my husband's cousin's neighbor's son much more often and I consider him as a real son to me. The objective dictionnary of the Who in general has no meaning in the study of emotional impact but the experience with that Who who will assess this impact.

How can one analyze the mathematical value of the Who emotional impact? What are the factors that make this Who impacting for me? Firstly the question that we all ask: Do I know that Who? If I know him, How much do I like/dislike him, how much did I interract with him or just hear about hin and how much do we share ? Otherwise, if I don't know him, Is the Who next to me right now and how much do we look like ?

Of course the both questions Is the Who next to me right now? and How much do we look like ? can be also integrated to the first type of the Who that I already know.

Five emotional impacts between the studied person $P$ and the Who are considered so far:

- The Relational Impact $I_{r}$ : How much do I like/dislike the Who?

- The Physical Impact $I_{p}$ How far is the Who next to me now?

- The Sharing Impact $I_{s}$ : How much do we share ?

- The Likelihood Impact $I_{l}$ : How much do we look like ?

- The Frequency Intensity $I_{f}$ : How many times did we hear about the Who indepently on its impact?

All these Who impact are summarized in the figure Fig.1.

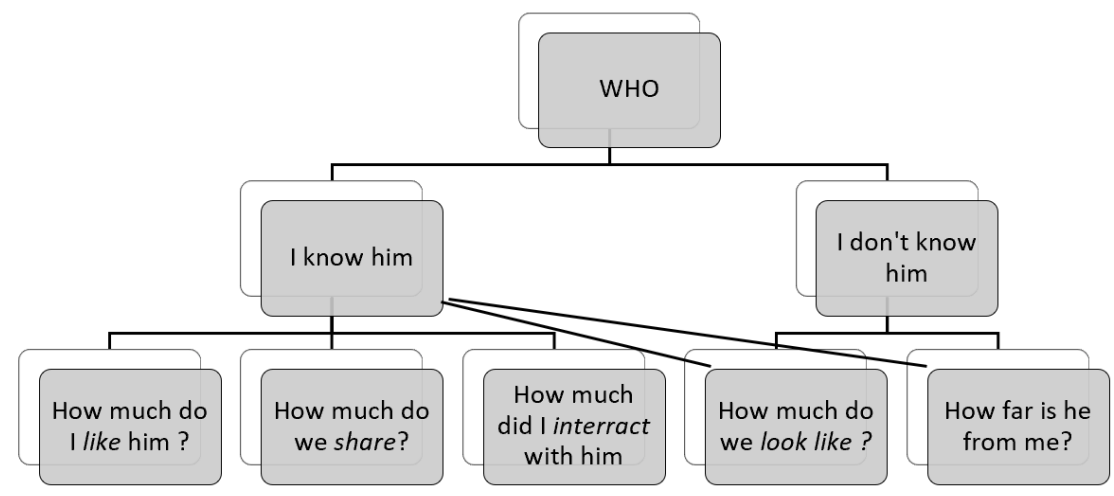

Fig. 1. The different emotional impact factors of the Who in the analyzed event to the stuied person

\section{A. The Relational Impact $I_{r}$}

Everyday we live with persons that we already know or we have never seen before. People or things that we already know by having social interaction with them (direct, indirect through social medias, general medias...) we really like them or not at all. What makes a person or a thing or a concept or anything be likable to me or not? 
The answer is all the past events that have more or less positively or negatively impacted me which include information, actions, experiences where the $W$ ho had been included. The more the Who impacted me positively by the past, the more it is likable to me and conversely.

The past lived with the Who and precisely the past emotional impacts of that Who lead at each moment to a new impact on me positively or negatively [14], [15]. The aggregation of all the past emotional impacts correlated to the $W$ ho before the occurence of the studied event at time $t$ will define a new measure that we call the relational impact $I_{r}(t, W h o, P)$ between the $W h o$ of the event and the studied person $P$. This relational impact is related to the emotional intensities previously experienced from the past events. The older the impacting event is, the more likely that its emotional impact will be attenuated (see section VIII). The parameter $I_{r}$ is higher in absolute value if the impact of $W$ ho on me in the past has been very intense (negatively or positively).

When an event occurs with a particular Who all that we remember and know from this Who sprung up and we suppose that we subconsciously we take into account only the events with the highest intensity that may be correlated to the present event.

Hence we suggest a mathematical formula for this impact as following:

$$
I_{r}(T, P, W h o)=\operatorname{sign}\left(I\left(P, \text { Event }_{\text {argmax }_{t} I\left(P, \text { Event }_{t}, T\right)}, T\right)\right) \times \max _{t \in[0, T]} \mid I\left(P, \text { Event }_{t}, T\right) \delta_{W h o}\left(\text { Event }_{t}\right) \mid
$$

With $T$ is the time of occurrence of the studied event (present). Event $t_{t}$ the event perceived by $P$ at the past time $t$ (assuming that a person cannot perceive two events strictly at the same time). $I\left(P\right.$, Event $\left._{t}, T\right)$ is the evolution intensity of the emotion felt at past time occurence $t$ until time $T$ of the studied individual $P$ (see section VIII). $\delta_{W h o}$ is the Dirac measure which is equal to 1 if the Who belongs to the past event Event $t_{t}$ at time $t$ and is zero otherwise. Since the emotional intensity can be negative, this impact is allowed to be negative. The more negative is this impact, the more negative is the relationship between the Who and the person $P$.

Note that the studied person could have never met or shared events with the Who but may be impacted from afar by this Who that may be a celebrity, a politician, a scientist, an actor...

So far this Who can be an individual that has never be seen before and nothing is known about him. We suggest therefore another impact which is the Physical Impact.

\section{B. The Physical Impact $I_{p}$}

It is possible that this $W$ ho of the event has never been seen before, but right now it is right next to the person studied $P$. This $W h o$, that I do not know is now experiencing an event should not impact me directly. However his physical distance to me matters. Hence this $I_{p}$ does not really exist because it is not about the $W$ ho in itself but in the physical distance which is included in the stimulus Where. For more details see section VI-B.

\section{The Sharing Impact $I_{s}$}

A Who could be something (an organization, a company, a contractor ...) that I may have never met but I share with it material things. The measure considered here is the proportion of what I 
own of this $W$ ho at time $T$ denoted by $\pi_{1}(t)$ : for example the $20 \%$ of my financial portfolio are shares of a company A (company A is the $W h o$ ), the sharing impact $I_{s}$ with company A will be linked to this 20\%. The greater is this proportion between the Who and the person studied, the higher is the intensity of the emotional impact. This impact could be formulated as follows:

$$
I_{s}(P, W h o)=\psi\left(\pi_{1}(t)\right)
$$

where $\psi$ function is a nondecreasing function to be defined.

\section{The Likelihood Impact $I_{l}$}

We add another measure of impact between the Who and the impacted person, which we will call the Likelihood Impact $I_{l}$. This measure, as well as the other three, can be either negative or positive. The Likelihood impact consists in comparing what define my identity to others at the present time such as my age, my gender, my nationality, my profession,... to the Who while supposing that this Who is a person. For example, the impact of the event "someone who is younger than me has managed to do an achievement that I have not been able to accomplish until now" is different from "someone Who is older than me has succeded what I haven't been able to accomplish so far ".

The impact of the first event is emotionally negative for me as it inspires a kind of regret that I haven't been able to accomplish by the past. While the impact of the second event could be positive and could give me hope. This impact tends to be positive if the age difference between the $W h o$ and the person studied is positive (the Who is older). Conversely, it will be negative, see figure Fig. 2 for the age comparison.

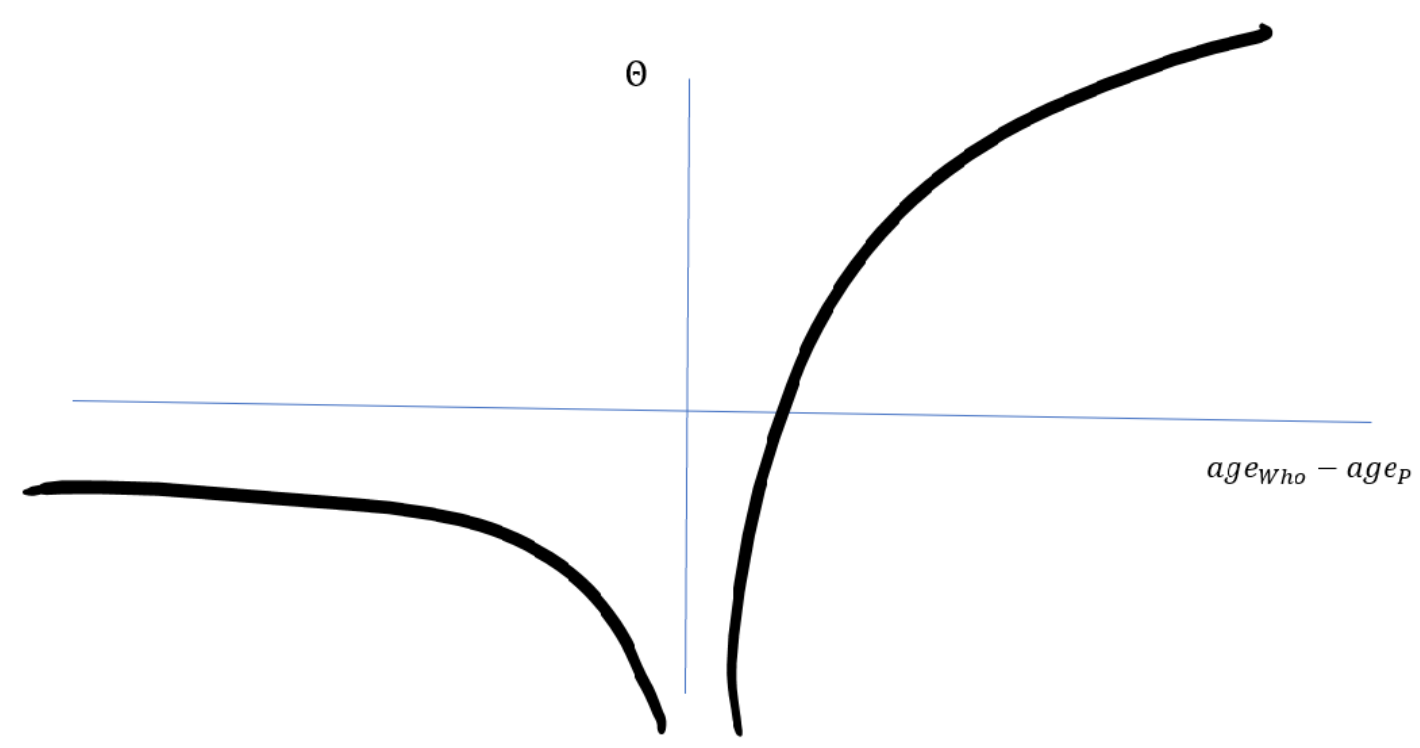

Fig. 2. Variation of the Likelihood age impact between the Who and the studied person $\mathrm{P}$ 
We formalize the impact of the age difference as follows:

$$
I_{l}(P, W h o)=\theta\left(a g e_{W h o}-a g e_{P}\right)
$$

In general case if we compare a same identity between the $W$ ho and the studied person $P$, the formula will be the same as follows:

$$
I_{l}(P, W h o)=\theta\left(\text { Identity }_{W h o}><_{P} \text { Identity }_{P}\right)
$$

Where Identity $y_{W h}$ is the identity of the Who of the event occured and Identity $y_{P}$ is the identity of the studied person. The symbol $><_{P}$ here means that we compare between the two Identities relatively to the studied person $P$. for example: being a doctor for a person $P_{1}$ may be more rewarding than being a researcher, in difference to a person $P_{2}$ which thinks the opposite?

The less is the difference between the identity of the $W h o$ and the person $P$ the higher is the emotional impact on the last one. The main raison is that we compare ourselves to persons who are similar to us.

We could also compare with other personal traits such as intelligence, strength, beauty, wealth... but we will see that these traits can be integrated into the formulation of the What.

\section{E. The Frequency Impact $I_{f}$}

Sometimes even we do not like some people/thing, we feel an unconscious sadness to lose them. The main reason for this sadness is none other than the habit of living with this Who. The frequency impact may be formulated as follows:

$$
I_{f}(W h o, T)=\frac{1}{T} \sum_{t<T} 1_{W h o \in E v e n t_{t}}
$$

This intensity sums only how much times a person $\mathrm{P}$ interracted/heard/saw... the Who before the occurence event time $T$.

Conclusion: These 5 Who emotional impact intensities will each have a different weight in the final emotion intensity of the studied Event which will depend on the emotion studied:

$$
Q i_{e}(P, W h o, t)=f\left(I_{r}(P, W h o), I_{p}(P, W h o), I_{s}(P, W h o), I_{l}(P, W h o)\right)
$$

\section{THE What MODEL: 3 VALUeS TYPE}

The Who makes an action to get/lose a What voluntarily or not. He can buy/sell this material or immaterial What. He can be assigned/withdrawn this What which can also be a qualifying adjective. The Who can succeed/fail to achieve the What. A What may be a person, an object, a concept, a project, a qualifying adjective, an competition, a company, a product, a material or immaterial good, a law, a right ... 
The What, like the Who despite its unchangeable definition in the dictionary, impacts us in different ways depending on the context of time, space, quantity, the manner and the reason (When, Where, How Much, How, Why).

The What is a valuable thing relatively to the impacted person $P$. Our goal is to model the value of the What without knowing a priori its own definition in the dictionary. Three different and independant values are considered in this study: the Rarity or the External Emotional value $v_{r}$, the Influence value $v_{i}$, and the relative Need value $v_{n}$.

\section{A. The External Emotional value of What: the rarity value $v_{r}$}

For a good that already has a price, the external emotional value of this good depends on its price which depends on the market, the supply and the demand [16], the usefulness of this good, the place where we buy it, the time when we take it, or can be modeled directly mathematically (financial derivative products for example) ... A house of $200 \mathrm{~m}^{2}$ has a different value if it is in the countryside or in Manhattan. It also has a different external emotional value whether it was bought 20 years ago or now.

The What can have no monetary price such as adjectives, concepts, examinations, facts, death, accidents ... How then to give an external emotional value to these concepts? We assume that this value depends on the number of demands and offers for this concept: for example the concept "lawyer exam" is valued by the probability of passing this exam: there are a certain number of candidates (the demand) for a certain number of places (the offer). But the probability of having this exam depends also on the year (number of candidates which can vary), on the place (France or USA or China...), on how the exam was passed (without the help of anyone or boosted), why (because the father of a candidate is one of the greatest lawyers in USA ...). This value identifies the intensity of the rarity of the What.

The more the What is rare or difficult to reach for the person studied $P$, the higher its value according to the external emotional value of the What. This external emotional value of the What can thus be negative or positive as it depends also on the sign of the difference between the number of demands and offers. If the offer exceeds demand, the What is considered undesirable and hence the $v_{r}$ is negative and conversely.

We formalize this value as follows:

$$
\begin{array}{r}
v_{r}(P, \text { What })=\xi\left(\operatorname{sign}\left(N_{D}-N_{O}\right) \times \mathbb{P}\left(\text { Achieve }_{P}=\text { What } \mid \text { Where, } . .\right.\right. \\
, \text { When, How Much, Why, How })) .
\end{array}
$$

With $\operatorname{sign}\left(N_{D}-N_{O}\right)$ is the sign of the difference between the number of demand and offer and of course the number of demand and offer, $N_{D}$ and $N_{O}$, is relative to what the studied person $P$ knows.

In addition, we add $\mathbb{P}\left(\right.$ Achieve $_{P}=$ What $\mid$ Where,...$)$ which is the probability of having the What in question relative to the person studied $P$ knowing the others stimuli Where, When, How Much, Why, How. The external emotional value of this What will therefore depend on these two elements according to a function $\xi$ which could have the form as shown in Figure Fig.3 . The lower the probability is, the higher the What has an external emotional value and vice versa. 


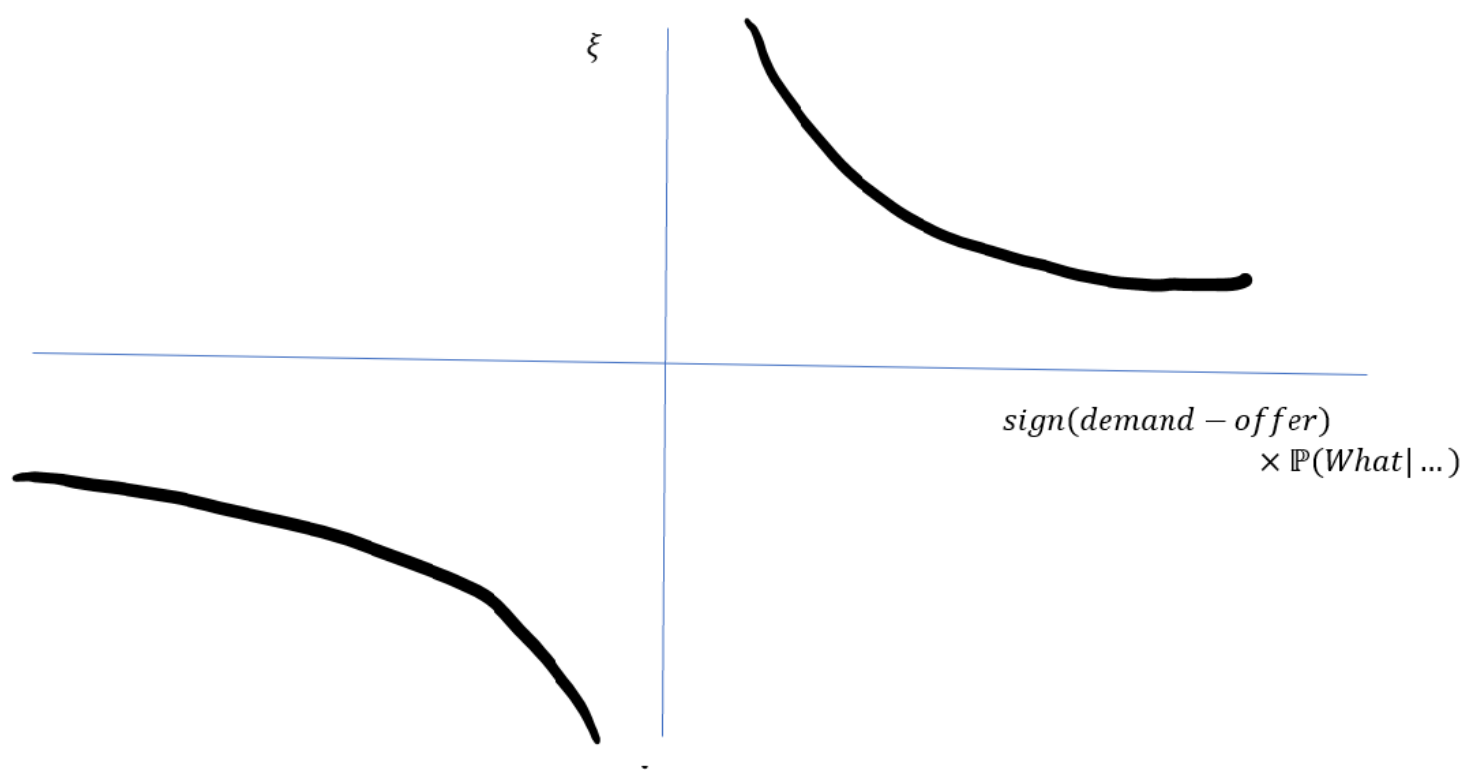

Fig. 3. Variation of the external or rarity value to the probability to gain the What and to the difference between demand and offer

\section{B. The Influence value $v_{i}$ : a social pressure value}

The influence value of a What depends on the number of times this What arises in previous events and the emotional impact intensity of these events. As stated in Maël LEBRETON's article [17], people want what others have and want, so the value of What increases more and more when others have this That. But if a person that I do not like wear a shirt that I was thinking to buy it, I would probably not buy it. This value does not evolve only by the number of people Who have this What but also by the relative impact of the What on me. "The house of $200 \mathrm{~m}^{2 "}$ in addition to having its own price which depends on the place and etc. (see formula 9, has an influence value $v_{i}$ which depends on the experience of the person studied $P$. Let us take the example of a What related to the following past events: "my sister bought a house 3 years ago", "my neighbor bought a house", "my brother's friend bought a house" ...

The What, which is "buying a house" in this example, would be more significant for the individual $P$ experiencing all these events at the same time comparing to the person who has not yet experiencing events involving "buying houses" before. The first person was strongly influenced by the events/information related to the home purchase. This valuation $v_{i}$ looks like the definition of the relational impact $I_{r}$ which depends on past impacts and on the number of times in which the What occurs and can be written samely as $I_{r}$ except one difference:

$$
\begin{array}{r}
v_{i}(T, P, W h o)=f_{i}\left(\operatorname{sign}\left(I\left(P, \text { Event }_{\text {argmax }_{t} I\left(P, \text { Event }_{t}, T\right)}, T\right)\right)\right. \\
\left.\times \max _{t \in[0, T]} \mid I\left(P, \text { Event }_{t}, T\right) 1_{d\left(\text { What }_{T}, \text { What }_{t}\right)<\gamma}\left(\text { Event }_{t}\right) \mid\right)
\end{array}
$$

Where $1_{d(\text { What,What })<\gamma}\left(\right.$ Event $\left._{t}\right)$ is the indicator function which is equal to 1 if the distance between the present $W h a t_{T}$ and the What $t_{t}$ attached to previous Event $t_{t}$ occured of time $t$ is less 
than a certain threshold $\gamma$ (distance to be defined), and is equal to zero otherwise. We therefore take into consideration all the intensities of past events where the previous What $t_{t}$ are in the equivalence class of the present What $t_{T}$. Example of a similar What: house, flat, villa, land... or an adjective What: beautiful, pretty, cute, charming...

\section{The relative Need value $v_{n}$ : a consumption value}

This value is linked to the quantity of What consummed recently and its consumption speed over time until its disappearance or expiration. This value can be also defined as the need degree of the What. The impact of seeing a cold bottle of water is not the same on a person that had already drunk water and on a person who did not drink anythink for 48 hours. The need value is subjective [18] and has to be taken into account to alleviate the diamond/water paradox [19]: "There is nothing more useful than water, but it can hardly buy anything [...] A diamond, on the contrary, has almost no value in terms of use, but we will frequently find it exchanged for a very large quantity of other goods. If in a desert, far from any water source and from anything else, if we give the choice to a person who did not drink water for 48 hours between a $10 \mathrm{~L}$ of water and $10 \mathrm{Kg}$ of diamonds, the decision is not even made.

We mentionned previously that the need of the What depends particulary on the speed of consumption and the initial quantity we own of the What, but also the frequency of the consumption during time. Supposing that the quantity of the What varies over time (due to consumption and frequency of consumption), the impact value of this What relatively to the person $\mathrm{P}$ depends on the last quantity consumption of that What and what is consummed until the occurence event time $T$. Example: what is the need value $v_{n}$,right now, of a liter of water without considering its price (its external value) nor its influence value (I have not seen people in front of me drinking water)? Its value therefore depends on the last consumption of water $C_{P}$ and especially on the desire now to drink water which depends on the amount of water remaining in my body $R(t, T)$, and finally on the relative frequency function in time of water consumption $f_{c}$. If normally, on average I drink a glass of water each two hours, in the next two hours my need of drinking water will increase event my body does not want it. This is the reason why we add the parameter frequency $f_{c}$ in this study.

We assume also that the more the frequency is higher and on average monoutonous in time the more the What is important and needed. Let $\zeta$ be a function describing the variation of the value $v_{n}$ depending on $C_{P}-R(t, T)$ that describes the remaining quantity of the What, and the frequency of consumption during time $f_{c}(t, T)$ from $t$ to now $T$ which is can be written as follows:

$$
v_{n}(P, \text { What }, t, T)=\zeta\left(C_{P}-R(t, T), f_{c}(t, T)\right)
$$

The lower is the quantity $C_{P}-R(t, T)$ the higher is the need value $v_{n}$. 


\section{QUANTITATIVE MODELS OF SOME EMOTIONS: A PERMANENT COMPARISON OF ONESELF AND WITH OTHERS IN PAST, PRESENT AND FUTURE}

In this section, we want to model each emotion with a different formula. The emotion with the higher intensity in absolute value will be the emotion felt at this moment with the highest probability.

Any emotion previously felt tends to fade over time. Moreover, a same event that occurs in two different times governs different emotions in terms of intensity. the main reasons are that humans learn over time and get used to any situation. Therefore to compute the emotion felt, it is necessary to deduce the part of "expectation and habit" resulted from previous situations wich can be formulated by a conditional expectation to what the studied person $P$ has experienced previsouly (see all formulas in this section) with a discount factor to fade the past intensity of the emotion felt.

\section{A. Angry/Guilty:}

The $W h o$, if different from the studied person $P$, is taking from the studied person $\mathrm{P}$ a $W h a t$ with a positive value or giving him a negative What. As a reminder, a What can be an object, a person, an adjective .... The studied person will be angry. Otherwise, if the studied person is taking from the Who a positive What (the aggregate What value is here negative), he may feel guilty.

The angriness therefore depends on how much valuable, in absolute value, has been taken. What we value here is the angriness relatively to the Who of the event. It depends on how much of the What has been taken by the $W$ ho from the person $P$ relatively to him which is denoted by $d V_{p}$ and is a function of $v_{r}, v_{i}, v_{n}(9,10,11)$, and on how much does the Who owns of the $W h a t V_{w}$. Knowing that the analyzed What for the studied event is the same for the Who and the concerned person $P$, one can rewrite:

$$
\begin{gathered}
V_{p}=K_{p} \times V \\
V_{w}=K_{w} \times V
\end{gathered}
$$

where $K_{p}$ is the quantity of the What that the individual $P$ owns and $K_{w}$ the quantity of this What that the Who have. $d V_{p}$ is the value of lost relatively to the person $P$.

We suppose that by losing $d V_{p}$, at the same time the $W h o$ in the other hand is gaining $d V_{w}$ a fraction of $d V_{p}$ that may be described by this formula : $d V_{w}=-K d V_{p}$ where $K$ is a positive factor. If $K>1$ therefore not even that person $P$ is giving to the $W h o$ but also other participants are involved and the anger should be alleviated and probably shared among these people. However if $K<1$ then what person $P$ is losing is shared between the $W h o$ and other involved participants and the anger could get higher as he would believe that the winners are ganging up against him.

The intensity of angriness/guilt depends also if I am used that the Who takes me that What frequently. I will not be as angry that my salary every month is lower than what I have been told because of different taxes ... because I know it is like that every month. The habit and the expectation is modeled as the conditionnal expected value knowing what the person $P$ experienced 
before of every past events while supposing that every emotion tends to fade during time (discount factor):

$$
I_{\text {Angry }}\left(P, \text { Event }_{T}\right)=\left(\left(\nu\left(K, d V_{p}, V_{p}, V_{w}\right)-\mathbb{E}\left(\nu\left(K, d V_{p}, V_{p}, V_{w}\right) \times \operatorname{discount} \mid \mathcal{F}_{T-1}\right)\right) \times 1_{d V_{p}<0}\right)_{+}
$$

and

$$
I_{G u i l t y}\left(P, \text { Event }_{T}\right)=\left(\left(\nu\left(K, d V_{p}, V_{p}, V_{w}\right)-\mathbb{E}\left(\nu\left(K, d V_{p}, V_{p}, V_{w}\right) \times \operatorname{discount} \mid \mathcal{F}_{T-1}\right)\right) \times 1_{d V_{p}>0}\right)_{+}
$$

where $\mathcal{F}_{T-1}$ is all the previous information and events before the occurence time $T$ of the studied event Event $_{T}$. The sign + in these two formulas means that we take the comptued value only if it is positive and otherwise it is equal to zero.

\section{B. Sadness and happiness:}

The studied individual succeded (happiness) or failed (sadness) to achieve a positive What of value $V$. The difference between the angriness and guiltness is that a person is feeling angry when a particular Who is taking from him something while he is feeling sad when he failed to achieve a goal withount necessarily another person taking it from him. Even if we are waiting for a gain we still be happy but less than if we have not expected that before:

$$
I_{\text {Happy }}\left(P, \text { Event }_{T}\right)=V \times\left(1_{V>0}-\mathbb{E}\left(1_{V>0} \times \operatorname{discount} \mid \mathcal{F}_{T-1}\right)\right)_{+}
$$

and

$$
I_{\text {Sad }}\left(P, \text { Event }_{T}\right)=V \times\left(1_{V<0}-\mathbb{E}\left(1_{V<0} \times \operatorname{discount} \mid \mathcal{F}_{T-1}\right)\right)_{-}
$$

where $1_{V>0}$ describes the case where there is success, and hence $\mathbb{E}\left(1_{V>0} \times\right.$ discount $\mid \mathcal{F}_{T-1}$ is the probability of success knowing all the anteriour information.

\section{Surprise and disappointment:}

Surprise or disappointment can be qualified as the unexpected gain or loss for the studied person $P$. Its intensity depends on the difference between the present value $V_{T}$ received of the What and the conditional expected value $V$ until the time before the event occurence $T-1$ wich is formulated by $\mathbb{E}\left(V \mid \mathcal{F}_{T-1}\right) . \mathrm{V}$ in this case is supposed to be random process in time and the intensity of the suprise or disappointement are described as follows:

$$
I_{\text {Surprise }}\left(P, \text { Event }_{T}\right)=\left(V_{T}-\mathbb{E}\left(V \mid \mathcal{F}_{T-1}\right)\right)_{+}
$$

and

$$
I_{\text {Disappointment }}\left(P, \text { Event }_{T}\right)=\left(V_{T}-\mathbb{E}\left(V \mid \mathcal{F}_{T-1}\right)\right)_{-}
$$

where the sign - in the last formula means that we take the comptued value only if it is negative and it is equal to zero otherwise. 
D. Envy:

The envy comes from the fact that the Who, supposing different from the person $\mathrm{P}$, is gaining relatively more than the studied person $P$ owns $V_{P}$ on the correlated What and even more than the Who did before. If the Who gains at each time the same amount $V_{T}$ of What then the intensity of envy is therefore attenued. It depends therefore on how much and how frequency does the Who gain this What with value $V_{T}$ and its equivalents:

$$
I_{\text {Envy }}\left(P, \text { Event }_{T}\right)=\left(\left(V_{T}-V_{P}\right)-\mathbb{E}\left(\left(V_{T}-V_{P}\right) \mid \mathcal{F}_{T-1}\right)\right)_{+}
$$

\section{E. Fear/Hope:}

Fear comes from the fact that there is a possibility of losing something valuable or gaining a negative valuable thing $O$ that we own with value $V_{p}(O)$ and has a correlation $c(O$, What $)$ with the What of the studied event. It is a kind of a loss expectation in future knowing all the information up to the present and giving future scenarios that person $P$ can forecast:

$$
I_{\text {Fear }}\left(P, \text { Event }_{T}\right)=\sum_{O \in \text { owns }} \frac{c(O, \text { What })}{C} \mathbb{E}\left(\frac{d V_{p}(O)}{d t} 1_{\frac{d V_{P}(O)}{d t}<0} \mid \mathcal{F}_{T}\right)
$$

The $1_{\frac{d V_{P}(\text { Event })}{d t}<0}$ ensures that only loss is taken into account. We are summing here all the possible weighted correlated loss with $C=\sum_{O \in \text { owns }} c(O$, What $)$ as if we compute an expected loss of all what the person $\mathrm{P}$ owns.

To model the intensity of Hope emotion, the one difference will come in the indicatrice function where we hope that there will be this time a gain:

$$
I_{\text {Hope }}\left(P, \text { Event }_{T}\right)=\sum_{O \in \text { owns }} C(O, \text { What }) \mathbb{E}\left(\frac{\left.d V_{p}(O)\right)}{d t} 1_{\frac{d V_{P}(O)}{d t}>0} \mid \mathcal{F}_{T}\right)
$$

\section{F. Stress:}

The studied person $\mathrm{P}$ does not know what to expect in the future following the occurence of the studied event. The more the What is hazardous, the higher is the stress. One can describe this emotion by the variance of the fluctuation of the future value $V$ of the What knowing information up to occurence time of the studied event defined by $\mathcal{F}_{T}$ :

$$
I_{\text {Stress }}\left(P, \text { Event }_{T}\right)=\operatorname{VAR}\left(v \mid \mathcal{F}_{T}\right)
$$

where $\operatorname{VAR}\left(. \mid \mathcal{F}_{T}\right)$ is the variance of the random variable conditinaly to the knowing information up to now at time $T$.

Other emotions could also be modeled mathematically. 


\section{THE Where MODEL}

Every event that occurs has its location this what we call here by the Where of the event. A Where can be described by distances with miles, meters.. or by a specific nominative place like streets, cities, countries...

An event that occurs physically next to me is obviously more impacting than an occuring event further. However sometimes even events occuring $10000 \mathrm{~km}$ further can be impactful. What are the impacting factors of the event's Where that influence my emotional state ?

\section{A. Where, an implicit parameter of the What}

As introduced in the section IV of the What definition, the What value depends on its external value (rarity, demand, supply...), its influence impact and its need degree. All these described values depend on the Where. An appartement of $200 \mathrm{~m}^{2}$ is more expensive in Manhattan (New York, USA) than in Detroit. Samely the value of the need for soap in France during the coronavirus period is much higher than in some African countries due to the low spread or absence of the virus in these countries. The Where and the When are both implicit impact factors of the What.

\section{B. The Physical distance $d_{p}$}

It is possible that the Who of the event is right next to me and he undergoes a significant impacting event, such as a happy event: lottery win, marriage proposal..., or a disastrous event: assault, accident..., this will also will impact me even if I have never know this Who. This since "it could have happened to me".

The effect of the proximity in physical distance has been demonstrated in several previous publications see for instance [20], [15] and [21]. Also this feeling due to the proximity has been described as a kind of contagion [22]. So the measure considered here is the physical impact distance $d_{p}$ which takes into consideration the real physical distance between the Who and the person studied $P$ (in meters or feet for example) in addition to a personal coefficient describing the degrees that te person studied $P$ puts himself in Who's shoes $\alpha_{P}$. The given formula of the Physical impact $d_{p}$ between the studied person $P$ and the Who of an analyzed event is:

$$
d_{p}(P, W h o)=\phi\left(\alpha_{P}(t), \operatorname{Dist}(P, W h o)\right)
$$

With $\phi$ is at least an exponential decreasing function and $\operatorname{Dist}(P, W h o)$ is the real distance in meters or feet (for example) between the studied person $P$ and the $W h o$. The greater is this distance, the lower is the impact of the analyzed occured event. 


\section{The Relational distance $d_{r}$}

in addition that the Who may be a place for example in an Event where a country, or precisely its government, has made certain decisions, a place is also a Where where an action or an event has or is or will occur. Besides the fact that a place is a matter of distances, it is also a matter of historical relations with the studied person $P$.

I could say "I kinda like Eiffel Tour but I prefer by far Champs Elysees". What makes me prefer Champs Elysees to Eiffel tour? The answer is samely as before, I had better experiences in Champs Elysees by the past.

The relational distance to the Where has to be integrated into the emotional impact of an event. This relational distance is similar to the relational impact $I_{r}$ described in section III-A. The formula is therefore a little different from equation (3):

$$
\begin{array}{r}
d_{r}(T, P, \text { Where })=\operatorname{sign}\left(I\left(P, \text { Event }_{\text {argmax }_{t} I\left(P, \text { Event }_{t}, T\right)}, T\right)\right) \times \\
\max _{t \in[0, T]} \mid I\left(P, \text { Event }_{t}, T\right) 1_{d\left(\text { Where }_{\text {Where }} \text { Event }_{t}\right)<\epsilon} \mid
\end{array}
$$

The main difference here from the formula (3) is the factor $1_{d\left(\text { Where, }_{\text {Where }} \text { event }_{t}\right)<\epsilon}$ which allows to take into account not just the precisely Where but also all the places of the past events that are very close by distance, maximum distance of $\epsilon$, to the Where of the studied event.

\section{THE WHEN MODEL}

\section{A. An information that complete the value of the What}

As for the Where which can be an implicit impacting factor VI-A, the When is also an implicit impact parameter in the What. See for instance the formula of the external value of the What 9. We reconsider the example of the soap during corona virus period. Six months before, the subjective value of the soap was in its price. There was no emergency need neither a great influence of the personal entourage that brings the subjective value of the soap higher.

The When which is one of the elements that describe the context is therefore one important parameter to determine the value of the What in the past, the present or the future.

\section{B. A probability that the studied event occurs}

Not all the events occured by the past or is occuring right now. Events may also occur in future. We can give examples: "the government has decided to increase taxes by $5 \%$ next year", "my son has decided to leave home in 4 years", "my son has decided to leave home tomorrow"...

The difference between the two last examples are the When, or the time that the event will occur. The second event that will occur tomorrow is more emotionally impacting than the one which will takes place in 4 years. What makes the difference? The only difference between these two examples is the distance in time from the present to the future When.

The same event, with the same elements, that may occur tomorrow is more probably to occur than the one that may occur in 4 years. This probability depends on the Who, What and all the 
other elements. The occurence probability of an event in a future When can be considered as the survival probability of the event until When time.

Let's take the first example introduced in the beginning " the government has decided to increase taxes by $5 \%$ next year", if the government of my country ensures all the time that its decisions and laws are enforced then there will be a high chance, to me, that this event will occur next year...and so I may decide to go on strike... Samely for the two other examples if my son is someone serious and everything he says he makes sure that it occurs. The possible occurence of an event by the future When is therefore governed by the fact that the event may be realistic: can the Who reaches that What at the time When ?

This first variable which describes the capacity of achieving a goal at When depends therefore of the credibility of the $W h o$, the value of the future reached What of the event and the historical of the speed of how the Who is reaching a subject $d V_{W}$ that is supposed here a random process with tendency calculated statistically.

In this section, we introduce two probabilities described in the following example:

"My son will eat the cake in three hours that I am cooking".

The Who here is my son, the What is eating cake that I am cooking of an initial value $v_{T}$ and the When is $T+3$ hours. What is the probability that it will occur knowing my son and other supposed future scenarios during these three hours that may occur and are independent of my son?

The scenarios that I give of my son : "He will eat candies between time $T$ and $T+3 h$ " with a high probability $p_{1}$, "He will not eat anything between time $t$ and $t+3 h$ " with probability $1-p_{1}$. The first probablity that we want to compute is the chance that the value of the cake to my son at time $T+3 h$, if he eats or not the candies, will be higher for him than my estimated value of the cake and hence eating the cake will occur at time $T+3 h$ with high probability.

The second probability that we want to compute takes into account the other independent scenarios for example : "my cake will be discuting" with probability $p_{2}$ "my cake will be overcooked" with probability $p_{3} \ldots$ then the second probability will calculate the chance that the future expected value of the cake $\mathbb{E}\left(v_{\text {When }} \mid\right.$ scenarios $\left._{P}\right)$, knowing my scenarios and their probabilities, outperform with a tolerance $\tau$ the present value of the cooking cake $v_{T}$. If it is the case the event "eating the cake" will occur with high probability.

When we project ourselves into the future, we as human, we have the capacity to create different possible scenarios that may change the direction of the occurence of the studied event. We do unconsciously integrate random processes which are future events or what we call commonly possible future scenarios that may stop or enforce the event occurence at the When. Each scenario introduced has its own occurence probability in time and of course it depends on our experiences and our knowledge of the context. The formulation of the probability of occurence of an event at time When may be described as following:

$$
\mathbb{P}\left(\text { Occurence }_{\text {When }}\right)=\mathbb{P}\left(\text { Achieving }_{\text {Cpty }} \mid \text { Who, What }, \text { When }\right) \times \mathbb{P}\left(\text { Occurence }_{\text {scenarios }}\right)
$$

Where $\mathbb{P}\left(\right.$ Achieving $_{C p t y} \mid$ Who, What, When $)$ is the probability that the Who achieve that What at the When. $\mathbb{P}\left(\right.$ Occurence|scenarios $\left._{P}\right)$ is the probability that the event occurs independently on the capacity of the Who and depends only on the relative (to the person $\mathrm{P}$ ) possible 
scenarios independent of the Who. These two probabilities (that we have already introduced in the previous example) are defined as follows:

$$
\mathbb{P}\left(\text { Achieving }_{\text {Cpty }} \mid \text { Who, What }, \text { When }\right)=\mathbb{P}\left(V_{w}+\int_{T}^{W h e n} d V_{u} \geq \mathbb{E}\left(v_{W h e n} \mid \mathcal{F}_{T}\right)\right)
$$

And

$$
\mathbb{P}\left(\text { Occurence }_{\text {scenarios }}\right)=\mathbb{P}\left(\mathbb{E}\left(v_{\text {When }} \mid \text { scenarios }_{P}\right)+\tau \geq v_{T}\right)
$$

The probability of the capacity achievement (27) computes the chance that the Who, knowing his capabilities to achieve things correlated to the What, and initially got a initial value $V_{w}$ correlated to the What, will succeed to achieve at least the expected future value of the What at the time When in the studied event Event $t_{t}$ knowing information from the past about the value of this What $\left.\left(\mathcal{F}_{t}\right)\right) . d V_{u}$ is the gain at time unit $u$ achieved that we sum between times $T$ and When.

The second probability (28) describes the chance that the expected value of the What at time When, knowing possible subjective scenarios to individual $P$ ( scenarios $_{P}$ ) that may occur between time $T$ and the time When, to be -with a tolerating $\tau$ - higher than the present value $v_{T}$ of the What. If the expected value in the future When is depreciated by negative subjective scenarios then the probability of occurence becomes lower.

\section{EMOTIONAL IMPACT INTENSITY EVOLUTION OF AN EVENT}

An emotion produced from a past event is amplified by future events that are correlated to this event but also by anterior memories boosted by any intern or extern event. This emotion intensity is attenuated by the interference of other uncorrelated emotional events or by correlated events of opposite sign.

Suppose that exist only two high correlated events (positively or negatively) Event E $_{1}$ and Event $_{2}$. Event Ev $_{1}$ the first occured event at time $t_{1}$ and Event $t_{2}$ the second occured event at time $t_{2}$. Between these two events other decorrelated events has occured. The variation of the

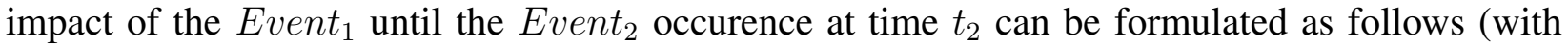
$I\left(\right.$ Event $\left._{2}, t_{2}\right)$ the instantaneous impact of Event $\left.{ }_{2}\right)$ :

$$
I\left(\text { Event }_{1}, t_{2}\right)=\frac{I\left(\text { Event }_{1}, t_{1}\right) M_{\text {Event }_{1}, \text { Event }_{2}}}{\sum_{k=t_{1}+1}^{t_{2}-1} I\left(\text { Event }_{k}, k\right) M_{\text {Event }_{k}, \text { Event }_{k+1}}}+\gamma\left(\text { Event }_{1}, \text { Event }_{2}\right) I\left(\text { Event }_{2}, t_{2}\right)
$$

where $M_{\text {Event }_{1}, \text { Event }_{2}}$ is the fading due to memory process between two events. Telecommunication experts may see that this formula is inspired from the model of received signal intensity in telecommunication field (Signal-to-interference and noise ratio SINR). At occurence time of Event $_{1}$ and before occurence of the second correlated event, other uncorrelated events more or less impacting arrived. This is a kind of interference signals that make the emotion intensity

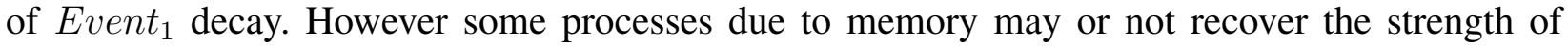


the emotion intensity caused by Event $t_{1}$ which is the fading $M_{\text {Event }}$, Event $_{2}$. When the second

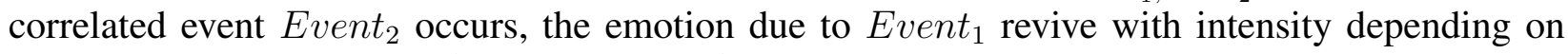
the degree of correlation $\gamma\left(\right.$ Event $_{1}$, Event $\left._{2}\right)$ between Event Ev $_{1}$ and Event $_{2}$.

The generalization of this formula if there are $N_{\text {corr }[t, T]}$ correlated events to the occured event Event $_{t}$ at time $t$ up to $T$ is as following:

$$
I\left(\text { Event }_{t}, T\right)=\sum_{i=1}^{N_{\text {corr }[t, T]}} \frac{\gamma\left(\text { Event }_{t}, \text { Event }_{c_{i}}\right) I\left(\text { Event }_{c_{i}}, c_{i}\right)}{\prod_{j=i}^{N_{\text {corr }-1}} \frac{1}{M_{\text {Event }_{j}, \text { Event }_{j+1}}} \sum_{k=c_{j}+1}^{c_{j+1}-1} I\left(\text { Event }_{k}, k\right) M_{\text {Event }_{k}, \text { Event }_{k+1}}}
$$

Where $\operatorname{corr}[t, T]$ are all the times between $t$ and $T$ where events are correlated to the Event $t_{t}$ and $N_{\operatorname{corr}[t, T]}$ is the number of the events correlated to Event $t_{t}$ from $t$ to $T$. Event $c_{i}$ is the $i^{\text {th }}$ correlated event from time $t$ to T. $\gamma\left(\right.$ Event $_{t}$, Event $\left._{c_{i}}\right)$ is a function of the probability that Event $_{c_{i}}$ may be connected to the studied Event $t_{t}$ by the brain. This parameter is a subjective parameter to each person and it describes how much past events are near to the analyzed event for the studied person. $M_{\text {Event }_{c_{i}} \text {,Event }}$ Evil $_{c_{i}}$ is the memory process occured between the Event $c_{c_{i}}$ and Event $t_{c_{i}+1}$. The denominator of the fraction of this sum is the interference due to other events between two correlated events.

The memory process $M$ is supposed to be a random process that considers a routing of more or less correlated memories noised by internal (other memories or psychological or physiological events) or external events. It behaves samely as the emotional intensities evolution equ.30 without adding other memory effects.

When an external event arrives, it also simulates old memories which are correlated to it ... and like a radio signal which is propagated by several paths (multipath) before being received by an antenna, the emotional signal due to the event will propagate in different trajectories involving several memories. The studied person may feel a different final emotion intensity: this is what we call the fading effect in telecommunication field. This final emotion intensity variable will follow Rice's distribution.

\section{CONCLUSION}

This study proposes a new way of how we could model some emotional elements of an event and no experimentation in this subject has been done yet. Future works to come will propose models linking all the stimuli in order to give an intensity of each possible emotion and subsequently deduce by mathematical modeling what type of behavior (optimized or unconscious or illogical) could result.

\section{REFERENCES}

[1] William James, Frederick Burkhardt, Fredson Bowers, and Ignas K Skrupskelis. The principles of psychology, volume 1. Macmillan London, 1890.

[2] Géraldine Coppin and David Sander. Théories et concepts contemporains en psychologie de l'émotion. 2010.

[3] Antonio R Damasio and R Descarte's Error. Emotion, reason and the human brain. New York: Putnam, 1994.

[4] Joseph Ledoux. Cognitive-emotional interactions: Listen to the brain. 2000.

[5] Lisa Feldman Barrett. How emotions are made: The secret life of the brain. Houghton Mifflin Harcourt, 2017. 
[6] Stanley Schachter and Jerome Singer. Cognitive, social, and physiological determinants of emotional state. Psychological review, 69(5):379, 1962.

[7] Tim Dalgleish and Mick Power. Handbook of cognition and emotion. John Wiley \& Sons, 2000.

[8] William A Cunningham and Philip David Zelazo. Attitudes and evaluations: A social cognitive neuroscience perspective. Trends in cognitive sciences, 11(3):97-104, 2007.

[9] Magda B Arnold. Emotion and personality (vols. i and ii). New York: Columbia University, 1960.

[10] Richard S Lazarus. Psychological stress and the coping process. 1966.

[11] Ainur Yessenalina, Yisong Yue, and Claire Cardie. Multi-level structured models for document-level sentiment classification. In Proceedings of the 2010 conference on empirical methods in natural language processing, pages 1046-1056. Association for Computational Linguistics, 2010.

[12] E Nikos, L Angeliki, P Georgios, and C Konstantinos. Els: A word-level method for entity-level analysis. In WIMS 2011 Proceedings of the International Conference on Web Intelligence, Mining and Semantics, 2011.

[13] Noura Farra, Elie Challita, Rawad Abou Assi, and Hazem Hajj. Sentence-level and document-level sentiment mining for arabic texts. In 2010 IEEE international conference on data mining workshops, pages 1114-1119. IEEE, 2010.

[14] Ian Burkitt. Social relationships and emotions. Sociology, 31(1):37-55, 1997.

[15] Lane Beckes and James A Coan. Social baseline theory: The role of social proximity in emotion and economy of action. Social and Personality Psychology Compass, 5(12):976-988, 2011.

[16] Jean-Baptiste Say. Traité d'économie politique. Calmann-Lévy, 1861.

[17] Maël Lebreton, Shadia Kawa, Baudouin Forgeot d'Arc, Jean Daunizeau, and Mathias Pessiglione. Your goal is mine: unraveling mimetic desires in the human brain. Journal of Neuroscience, 32(21):7146-7157, 2012.

[18] Carl Menger. Principes d'économie (grundsätze der volkswirthschaftslehre), 1871.

[19] Smith Adam. Recherches sur la nature et les causes de la richesse des nations. Economica, 1776.

[20] Daniel Knowles, Kyle Brown, and Silvio Aldrovandi. Does the proximity effect occur through non-consious processes? a study protocol. 2018.

[21] Bert Weijters, Maggie Geuens, and Niels Schillewaert. The proximity effect: The role of inter-item distance on reverse-item bias. International Journal of Research in Marketing, 26(1):2-12, 2009.

[22] Adam Maxwell Sparks, Daniel MT Fessler, and Colin Holbrook. Elevation, an emotion for prosocial contagion, is experienced more strongly by those with greater expectations of the cooperativeness of others. PloS one, 14(12), 2019. 